\title{
Does "cerebellar liponeurocytoma" always reflect an expected site? An unusual case with a review of the literature
}

\author{
Pinar Karabagli ${ }^{1}$, Aydin Sav², Necmettin Pamir ${ }^{3}$ \\ ${ }^{1}$ Department of Pathology, School of Medicine, Selcuk University, Konya, ${ }^{2}$ Department of Pathology, School of Medicine, Acibadem \\ University, i̇stanbul, ${ }^{3}$ Department of Neurosurgery, School of Medicine, Acibadem University, i̇stanbul, Turkey
}

\begin{abstract}
A rare tumour, cerebellar liponeurocytoma, is classified into glioneuronal tumours under the 2000 World Health Organization (WHO) classification of tumours of the central nervous system. The current 2007 WHO classification, therefore, assigns grade II to the cerebellar liponeurocytoma. Tumours are predominantly localized in cerebellar hemispheres, and the second most common location is the vermis. To date, approximately 40 reported cases of cerebellar and 10 cases of supratentorial intraventricular liponeurocytoma have been reported. In this report, an unusual case of cerebellar liponeurocytoma was presented with extracerebellar location. In the future tumour classification, it should be considered that liponeurocytomas are not restricted only to the cerebellum, but they are located in supratentorial areas as well.
\end{abstract}

Key words: liponeurocytoma, cerebellar, supratentorial, intraventricular.

\section{Introduction}

Cerebellar liponeurocytomas have been included in the 2000 World Health Organization (WHO) classification of tumours of the central nervous system, under the heading of glioneuronal tumours. It is a rare cerebellar neoplasm of adults with consistent neuronal, variable astrocytic and focal lipomatous differentiation, and with low proliferative potential. The current 2007 WHO classification therefore assigns cerebellar liponeurocytoma to WHO grade II [10,11].

In 1978, Bechtel et al. reported a case of lipomatous medulloblastoma in a 44-year-old man. The terms neurolipocytoma, medullocytoma, lipomatous glioneurocytoma, lipidized mature neuroectodermal tumour have also been proposed $[4,10]$. As a term, cerebellar liponeurocytoma is now largely accepted and is supported by genetic analyses that indicate that this lesion is not a variant of medulloblastoma $[7,10]$.

Tumours are predominantly located in cerebellar hemispheres, followed by a more central location in the vermis [10]. Tumours with features of liponeurocytoma have also been observed in supratentorial locations. There have been approximately 40 reported cases of cerebellar and 10 cases of supratentorial liponeurocytoma in the literature $[2,4-6,9,11,13,15]$. The tumour usually has a favourable clinical prognosis, although recurrences are frequent $[3,8]$.

We report a case of this tumour with supratentorial location. Characteristic features of these tumours are discussed in the light of pertinent literature. 


\section{Case report}

A 34-year-old previously healthy man developed a progressive headache. The neurological examination was normal. Magnetic resonance imaging (MRI) scan showed a large partly cystic, $4 \times 5 \mathrm{~cm}$ mass in the third ventricle (Fig. 1A). Enhancement with gadolinium was heterogeneous (Fig. 1B). The patient underwent an occipital craniotomy and gross total excision of the tumour. His postoperative period was uneventful.

The hematoxylin and eosin (H\&E) stained paraffin sections showed a moderately cellular tumour showing uniform, round oligodendroglia like cells containing round, "salt-pepper" nuclei and clear cytoplasm, and focal lipidized cells that comprised almost 10$15 \%$ of the tumour area. Upon detailed microscopic evaluation, these lipidized cells share some morphological consistencies with lipidized medulloblastoma and clear cell ependymoma (Fig. 2A). Only few mitoses and minute foci of micronecrosis were observed in the neuronal component. In immunohistochemical analysis neuron-specific enolase (NSE) (Fig. 2B) and synaptophysin (Fig. 2C) and MAP-2 immunopositivity were detected. Glial fibrillary acidic protein (GFAP), S-100, neurofilament (NF), vimentin, chromogranin, p53, EMA and desmin were immunonegative. Ki-67/ MIB 1 antibody immunolabeling index was 1.5\% (Fig. 2D). No neuroradiologic or clinical neurologic evidence of recurrence and/or residual tumour was noticed during a 2-year period of follow-up.

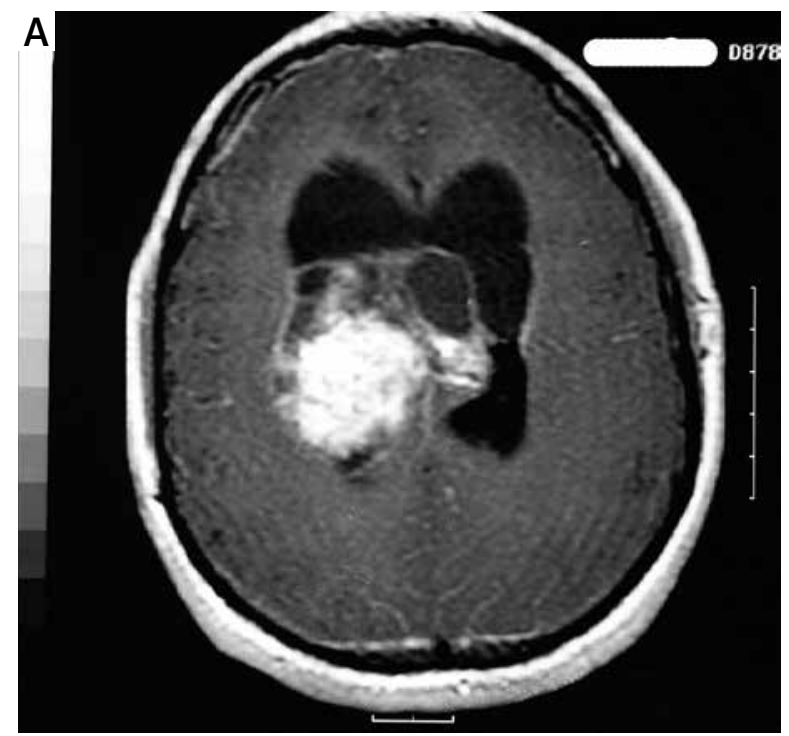

\section{Discussion}

Liponeurocytomas are rare cerebellar neoplasms with benign histological features and a favourable clinical prognosis. However, the current clinical opinion is based on a total of approximately 40 published cases $[8,10,14]$. Linking the concept of liponeurocytoma to its occurrence in the cerebellum unnecessarily obscures the existence of similar neoplasms at other sites. Indeed ten such cases have been reported in the English literature (Table I) $[2,4-6,9,11,13,15]$. We herein present the eleventh example of supratentorial intraventricular liponeurocytoma (Table I).

Liponeurocytomas are characterized by presence of various lipidized cells in clusters or scattered between small neoplastic cells. Immunohistochemical staining demonstrated both neuronal and glial differentiation. Histologically mitotic activity and proliferation rate are generally low in these lesions $[2,10,14,16]$.

The immunostaining profile of previously published cases of supratentorial liponeurocytomas demonstrates uniform reactivity for neuronal markers like synaptophysin, and/or NSE as was seen in the present case $[2,4-6,9,11,13,15]$. Expression of GFAP and S-100 is limited to scattered reactive astrocytes [4,9,15], and a few tumour cells $[2,5,11,13]$. The tumour cells are negative for NF $[2,4,5,9,11,15]$. MIB-1 labelling index was < 1\% [2], 4\% [11], 5.8\% [4] and $15 \%$ and increased to $30 \%$ in the recurrent tumour [9]. It was $1.5 \%$ in the present case and no

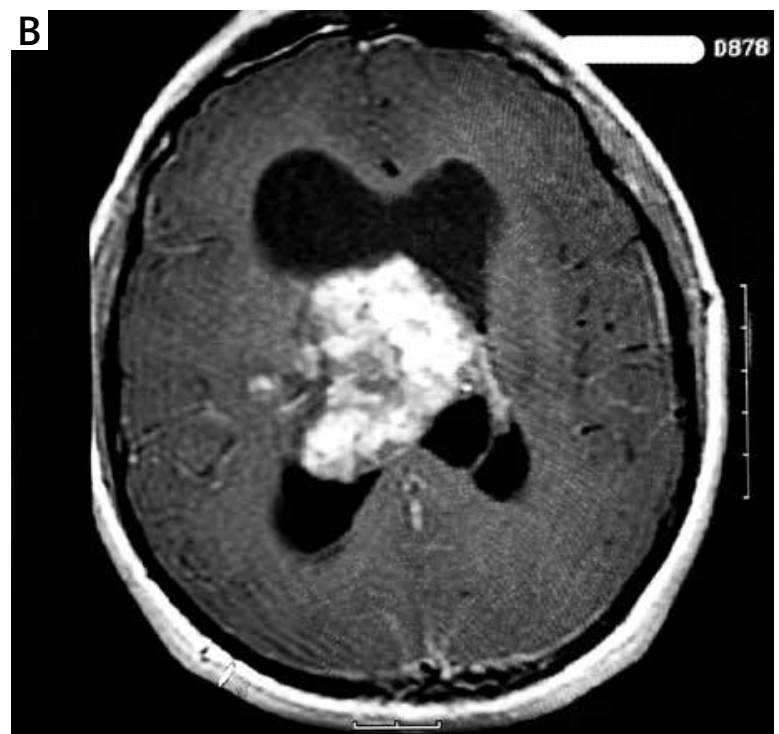

Fig. 1. On T1-weighted MRI showed a large, partly cystic, $4 \times 5 \mathrm{~cm}$ mass in the third ventricle with heterogeneous enhancement with gadolinium. 

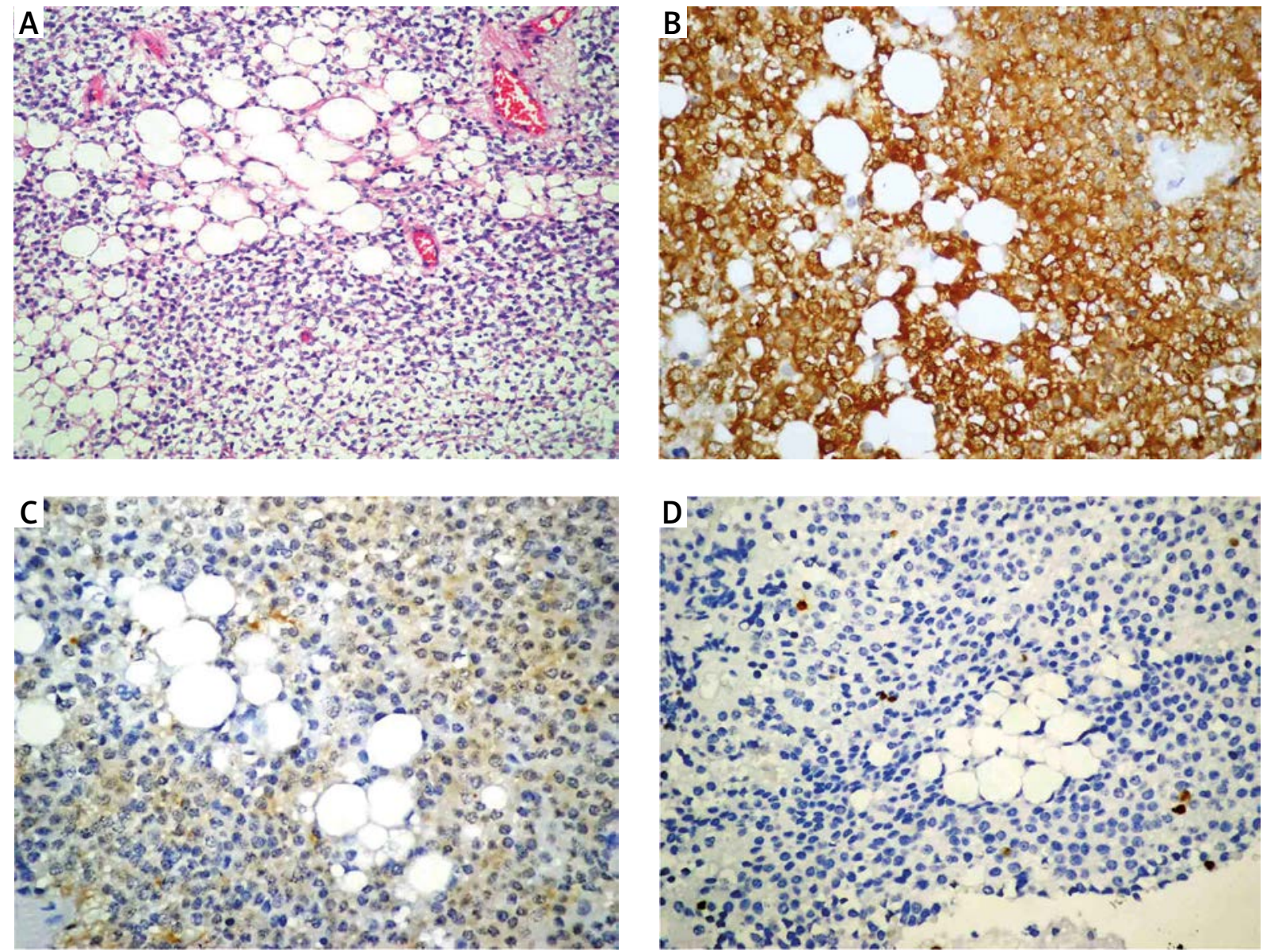

Fig. 2. A) Monotonous tumour cell proliferation with focal lipidized cells $(H E \times 200)$. B) Tumour cells reacting intensely with NSE (Anti-NSE; streptavidin-biotin complement, $\times 400$ original magnification). C) Synaptophysin reactivity is only focal and mild (Anti-synaptophysin; streptavidin-biotin complement, $\times 400$ original magnification). D) Ki-67/MIB-1 labelling index is low (1.5\%) (MIB-1; streptavidin-biotin complement, $\times 400$ original magnification).

recurrences were noticed during 2 years' follow-up period.

Among the relevant cases in files, only three of them were examined ultrastructurally. This particular consistent evidence provides that lipid vacuoles progressively accumulate and coalesce within cells while retaining their neurocytic features. Thus, these distinctive lesions are a result of tumoral lipidization rather than true adipose metaplasia $[4,5,9]$. Some authors have interpreted this lipidization as true adipose metaplasia [15]. Unfortunately, no ultrastructural study was applied to the case under investigation.

The immunohistochemical panel showed evidence of neuronal expression. Moreover, no immunoreactivity in lipidized tumour component was seen for synaptophysin, GFAP and S-100. We could not definitively confirm the presence of lipids within the cytoplasm of the tumour cells. Hypothetically, presence of lipid vacuoles most likely suggests the possibility of mesenchymal differentiation in the tumour [12]. The association of mesenchymal phenotypes in the tumour implies the possibility of a common origin from pluripotent cells related to such structures. Conveniently, this tumour may have derived from pluripotential embryonic ectomesenchymal stem cells of neural crest probably persisting in the ventricular matrix and the external granular layer $[1,6,12]$.

Apart from the cerebellar and 10 cases of supratentorial liponeurocytoma, it should be emphasized that the fact about lipidization within the neuroec- 
Table I. Summary of the 10 cases of supratentorial liponeurocytoma reported in the literature and our case

\begin{tabular}{|c|c|c|}
\hline Authors, [Ref. No] & Sex/Age (yr) & Tumor location \\
\hline Horoupian, 1997 [6] & $\mathrm{M} / 30$ & Left Lat v., $3^{\text {rd }}$ v., CC \\
\hline George, 2001 [4] & $F / 59$ & $\begin{array}{c}\text { Anterior horn, } \\
\text { Left Lat v. }\end{array}$ \\
\hline Rajesh, 2003 [15] & $\mathrm{M} / 30$ & Frontal horn, Lat v. \\
\hline Jouvet, 2005 [9] & $\mathrm{F} / 4$ & $4^{\text {th }} v$. \\
\hline $\begin{array}{l}\text { Kuchelmeister, } \\
2006 \text { [11] }\end{array}$ & $M / 35$ & Left lat v. \\
\hline Pankaj, 2010 [13] & $M / 35$ & $\begin{array}{l}\text { Lateral horn, } \\
\text { Right Lat v. }\end{array}$ \\
\hline Gupta, 2011 [5] & $F / 45$ & Trigone of Lat. v. \\
\hline Chakraborti, 2011 [2] & $\begin{array}{l}M / 36 \\
M / 30 \\
M / 32\end{array}$ & $\begin{array}{l}\text { Bilateral Lat v. } \\
\text { Lateral and } 3^{\text {rd }} \mathrm{v} \text {. } \\
\text { Bilateral Lat v., central }\end{array}$ \\
\hline (present case) & $M / 34$ & $3^{\text {rd }} v$. \\
\hline
\end{tabular}

todermal tumours of the central nervous system includes cerebellar astrocytomas, multiple intraspinal low grade astrocytoma, frequently in pleomorphic xanthoastrocytoma, occasionally in glioblastoma, ependymomas and supratentorial PNET [2].

Cerebellar liponeurocytoma has a relatively benign clinical course and a recurrence may appear after a long period of time [9]. The 5-year survival rate of cerebellar liponeurocytoma is $48 \%$ but this should be interpreted with caution because of rarity of this tumour and lack of systemic follow-up [10].

Cluster analysis of the cDNA expression data of 1176 genes grouped cerebellar liponeurocytomas close to central neurocytomas, but distinctive from medulloblastoma. Furthermore, the cDNA expression array data suggest a relationship to central neurocytomas, but presence of TP53 mutations (20\%), which are absent in central neurocytomas, suggest that their genetic pathways are different. Horstmann et al. reveal TP53 missense mutation with a higher frequency than medulloblastoma. Moreover, isochromosome 17q, a genetic hallmark of cerebellar medulloblastoma was not observed in any of the cases investigated in this study [7,10].

Total resection is considered the best possible treatment. There is no consensus regarding the specific treatment of liponeurocytoma, if only chemoor radiotherapy is an indispensable part of the postoperative treatment regimen $[3,8,10,14]$.

\section{Conclusions}

In conclusion, this study constitutes the $11^{\text {th }}$ case of "cerebellar" liponeurocytoma in an extracerebellar location. The future tumour classification should consider that liponeurocytoma is not restricted to the cerebellum, but may be located in extracerebellar sites as well. Consequently, it may be expected that the prefix 'cerebellar' would be omitted as evidenced by sporadic cases in the pertinent literature.

\section{References}

1. Akhaddar A, Zrara I, Gazzaz M, Moustarch d BE, Benomar S, Boucetta M. Cerebellar liponeurocytoma (lipomatous medulloblastoma). J Neuroradiol 2003; 30: 121-126.

2. Chakraborti S, Mahadevan A, Govindan A, Yasha TC, Santosh V, Kovoor JME, Ramamurthi R, Alapatt JP, Hedge T, Shankar SK. Supratentorial and cerebellar liponeurocytomas:report of four cases with review of literature. J Neurooncol 2011; 103: 121-127.

3. Gallina P, Buccoliero AM, Prillo V, Pansini G, Di Lorenzo N. Cerebellar liponeurocytoma: an update follow-up of a case presenting histopathological and clinically aggressive features. Neurol India 2009; 57: 194-196.

4. George DH, Scheithauer BW. Central liponeurocytoma. Am I Surg Pathol 2001; 25: 1551-1555.

5. Gupta K, Salunke P, Kalra I, Vasishta RK. Central liponeurocytoma: case report and review of literature. Clin Neuropathol 2011; 30: $80-85$

6. Horoupian DS, Shuster DL, Kaarsoo-Herrick M, Shuer LM. Central neurocytoma: One associated with a fourth ventricular PNET/Medulloblastoma and the second mixed with adipose tissue. Hum Pathol 1997; 28: 1111-1114.

7. Horstmann S, Perry A, Reifenberger G, Giangaspero F, Huang H, Hara A, Masuoka J, Rainov NG, Bergmann M, Heppner FL, Brandner S, Chimelli L, Montagna N, Jackson T, Davis DG, Markesbery WR, Ellison DW, Weller RO, Taddei GL, Conti R, Del Bigio MR, Gonzale-Campora R, Radhakrishnan W, Soylemezoglu F, Uro-Coste E, Qlan J, Kleihues P, Ohgaki H. Genetic and expression profiles of cerebellar liponeurocytomas. Brain Pathol 2004; 14: 281-289.

8. Jenkinson MD, Bomsa JJ, Du Plessis D, Ohgaki H, Kleihues P, Warnke P, Rainov NG. Cerebellar liponeurocytoma with an unusually aggressive clinical course: case report. Neurosurg 2003; 53: 1425-1427.

9. Jouvet A, Lellouch-Tubiana A, Boddaert N, Zerah M, Champier J, Fevre-Montange M. Fourth ventricle neurocytoma with lipomatous and ependymal differentiation. Acta Neuropathol (Berl) 2005; 109: 346-351.

10. Kleihues P, Chimelli L, Giangaspero F, Ohgakin H. Cerebellar liponeurocytoma. In: Louis DN, Ohgaki H, Wiestler OD, Cavenee WK (eds.).WHO Classification of Tumors of the Central Nervous System. IARC, Lyon 2007; pp. 110-112.

11. Kuchelmeister K, Nestler U, Siekmann R, Schachenmayr W. Liponeurocytoma of the left lateral ventricle-case report and review of the literature. Clin Neuropathol 2006; 25: 86-94. 
12. Mena H, Morrison AL, Jones RV, Gyure KA. Central neurocytomas express photoreceptor differentiation. Cancer 2001; 91: 136-143.

13. Pankaj R, Jindal A, Banerjee AK. Liponeurocytoma of lateral ventricle. Neurol India 2010; 58: 805-806.

14. Patel N, Fallah A, Provias J, Jha NK. Cerebellar liponeurocytoma. Can J Surg 2009; 52: E117-E119.

15. Rajesh LS, Vasishta RK, Chhabra R, Banerjee AK. Case report: central liponeurocytoma. Neuropathol Appl Neurobiol 2003; 29: 511-513.

16. Soylemezoglu F, Soffer D, Onol B, Schwechheimer K, Kleihues P. Lipomatous medulloblastoma in adults. A distinct clinicopathological entity. Am J Surg Pathol 1996; 20: 413-418. 